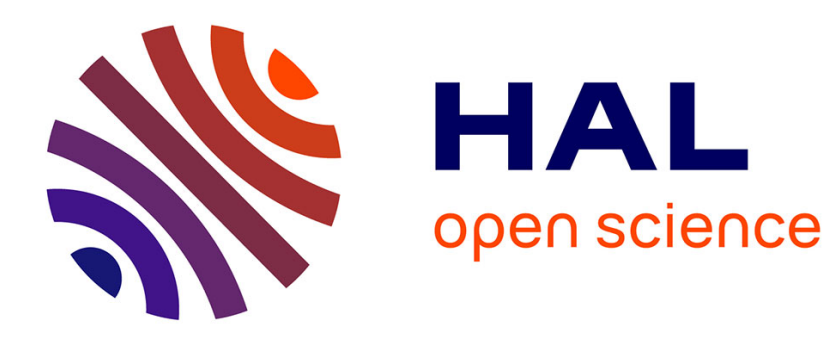

\title{
Smoothness of weak solutions of the spatially homogeneous Landau equation.
}

\author{
Mouhamad El Safadi
}

\section{To cite this version:}

Mouhamad El Safadi. Smoothness of weak solutions of the spatially homogeneous Landau equation.. 2006. hal-00023086

\section{HAL Id: hal-00023086 https://hal.science/hal-00023086}

Preprint submitted on 19 Apr 2006

HAL is a multi-disciplinary open access archive for the deposit and dissemination of scientific research documents, whether they are published or not. The documents may come from teaching and research institutions in France or abroad, or from public or private research centers.
L'archive ouverte pluridisciplinaire HAL, est destinée au dépôt et à la diffusion de documents scientifiques de niveau recherche, publiés ou non, émanant des établissements d'enseignement et de recherche français ou étrangers, des laboratoires publics ou privés. 


\title{
Smoothness of weak solutions of the spatially homogeneous Landau equation
}

\author{
Mouhamad EL SAFADI* \\ MAPMO UMR 6628 \\ Université d'Orléans, BP 6759 \\ 45067 ORLÉANS Cedex 2, FRANCE
}

February 1, 2006

\begin{abstract}
For the homogeneous Landau equation, we prove regularization properties of the weak solutions $f(v, t)$ and in particular that it lies in Schwartz space $\mathcal{S}$. This regularity was already obtained by Desvillettes and Villani [9] using weighted Sobolev spaces. However, our proof applies to any suitable weak solution, is more elementary and is based on Littlewood Paley decomposition of the velocity space into annulus parts. Apart from this decomposition, we only use elementary estimates, such as Cauchy-Schwarz or Young's inequalities.
\end{abstract}

\section{Introduction}

Landau equation is an important kinetic collisional model used to describe the time evolution of a system of particles in plasma physics (see [15] for instance). Particles are described through a distribution function $f(t, x, v)$ depending on time $t$, particle position $x \in \mathbb{R}^{N}$, and their velocity $v \in \mathbb{R}^{N}(N \geq 2)$. In this paper, we shall be exclusively concerned with the homogeneous case where $f(t, x, v)$ does not depend on $x$. In this case, the model writes as

$$
\frac{\partial f}{\partial t}(v, t)=Q(f, f)(v, t)
$$

*mouhamad.el_safadi@univ-orleans.fr 
where the initial datum $f(0, v)=f_{0}(v)$ is a given non negative function.

Landau quadratic operator $Q$ depends on v as follows:

$Q(f, f)(v, t)=\frac{1}{2} \sum_{\omega, \omega^{\prime}=1}^{N} \frac{\partial}{\partial v_{\omega}}\left\{\int_{\mathbb{R}^{n}} d v_{*} a_{\omega \omega^{\prime}}\left(v-v_{*}\right)\left[f\left(v_{*}, t\right) \frac{\partial f}{\partial v_{\omega^{\prime}}}(v, t)-f(v, t) \frac{\partial f}{\partial v_{* \omega^{\prime}}}\left(v_{*}, t\right)\right]\right\}$,

with

$$
\left(a_{\omega \omega^{\prime}}(z)\right)_{1 \leq \omega, \omega^{\prime} \leq N}=\Lambda|z|^{2} \chi(|z|) \Pi_{\omega \omega^{\prime}}(z) .
$$

Here, we shall always use the convention of implicit summation over repeated indices $\left(\omega, \omega^{\prime}\right)$. Above, $\Lambda$ is some positive constant that we shall normalize to be $1, \chi$ is a non negative function, and $\Pi(z)$ is the following non negative symmetric matrix, corresponding to the orthogonal projection onto $z^{\perp}$, i.e

$$
\Pi_{\omega \omega^{\prime}}(z)=\delta_{\omega \omega^{\prime}}-\frac{z_{\omega} z_{\omega^{\prime}}}{|z|^{2}}
$$

Landau collision operator (1.2) has the fundamental properties of preserving mass, momentum and energy, that is

$$
\int_{\mathbb{R}^{N}} Q(f, f) \phi(v) d v=0, \text { for } \phi(v)=1, v,|v|^{2} .
$$

Moreover, it satisfies the well known Boltzman's $H$ theorem, which writes formally as

$$
\int_{\mathbb{R}^{N}} Q(f, f) \log (f) d v \leq 0
$$

Arsen'ev and Buryak [1] have shown that solutions of the Boltzmann equation converge towards solutions of the Landau equation when grazing collisions prevail, while the full non homogeneous case was considered by Alexandre and Villani [5]. On this topic, one can consult the review paper of Villani [24], giving a lot of references.

We shall assume that the function $\chi$, entering Landau operator, is smooth and satisfies

$$
\begin{gathered}
c\left(1+|z|^{2}\right)^{\frac{\gamma}{2}} \leq \chi(|z|) \leq C_{0}\left(1+|z|^{2}\right)^{\frac{\gamma}{2}}, \\
\left|\nabla^{\beta}(\chi(|z|))\right| \leq C_{\beta}\left(1+|z|^{2}\right)^{\frac{\gamma}{2}-\beta} \text { for all integer } \beta,
\end{gathered}
$$

where $c$ is a non negative constant and $C_{\beta}$ a non negative constant depending on $\beta$.

Furthermore, we shall restrict herein to so-called hard potentials, meaning that the parameter $\gamma$ is in the range $0<\gamma \leq 1$. However, note that this assumption could be relaxed to $\gamma \leq 2$, 
and even to any $\gamma>0$, if we make some minor changes on the initial datum.

We shall also consider so-called very hard potentials, that is

$$
\chi(|z|)=|z|^{\gamma} \text { for } 0<\gamma \leq 1 \text {. }
$$

This case corresponds to interactions with inverse s-power forces for $s>2 N-1$. It differs slightly from (1.4), due to the singularity around zero.

Relevant existence results in the homogeneous case for weak solutions of (1.1) were obtained in $[1,7,9,10,11,22]$. A probabilistic framework is also worked out by Meleard and Geurin $[12]$.

In all the paper, we shall assume that a weak solution to Landau (1.1) has been constructed and that it satisfies the usual entropic estimate, for a fixed $T>0$ (eventually $T=+\infty$ )

$$
\sup _{t \in[0, T]} \int_{\mathbb{R}^{N}} f(t, v)\left(1+|v|^{2}+\log (1+f(t, v)) d v<\infty .\right.
$$

Let us mention that for the non homogeneous case, very few results are available, see [14, 25], and this is so even for the linearized equation $[6,18]$.

In this work, we are interested in regularization properties of weak solutions of (1.1). Such results were first shown by Arsen'ev and Buryak [1] for smooth and strongly decreasing initial data. Then, Villani studies it for Maxwellian molecules in [23]. The recent up to date results about this regularization question are due to Desvillettes and Villani [9], showing regularity in $\mathcal{S}$, for not necessarily smooth initial data. However, they needed conservation of all moments of $f$ to get this regularization property. But, according to their results, moments on the initial data are propagated along the solution flow and most importantly, they have also shown immediate appearance of higher moments, demanding only few ones on the initial data.

Herein, in order to simplify the exposition, we shall assume these moments estimates in $L^{1}$ space on the initial data, and thus on solutions itself.

Our goal in this paper is to obtain this $\mathcal{S}$ regularity, using only very elementary arguments. In particular, we avoid any use of interpolation inequalities, and most importantly, our method applies to any (suitable) weak solutions of Landau equation (1.1). Our method is based on Littlewood-Paley harmonic decomposition.

In a recent work, we have already used this method to prove very simply the immediate regularity of weak solutions of the homogeneous Boltzmann equation for Maxwellian molecules [3], showing an optimal $C^{\infty}$ regularity for all time $t>0$. The general case of non Maxwellian molecules is the subject of a work in progress [4]. Similar harmonic ideas are also used in 
[2], in order to get precise functional properties of a linear operator linked with Boltzmann quadratic operator, which can be used for instance to construct weak solutions for the full non homogeneous Boltzmann equation. In fact, in all these works, the main point is to work on small annuli instead of the full frequency space and otherwise we only use truly elementary analytical arguments.

Basic results about related harmonic analysis ideas can be found in the books of Runst, Sickel, Stein or Triebel [17, 20, 21].

Before stating our main result, let us firstly point out the basic assumptions and notations used herein.

Propagation of moments: The propagation of moments of solutions of (1.1)-(1.2) is proven in [9]. According to their results, we shall assume that

$$
\text { For all } s>0 \text {, if }\left\|f_{0}\right\|_{L_{s}^{1}}<+\infty \text {, then } \sup _{t \geq 0}\|f(t)\|_{L_{s}^{1}}<+\infty \text {. }
$$

As shown by Desvillettes and Villani, there is in fact immediate appearance of higher moments. It is possible to adapt the arguments of our work in this case too.

Formulation: In a standard way, other formulations of (1.1) and (1.2) are possible. For this purpose, we define, for $z \in \mathbb{R}^{N}$

$$
\left\{\begin{array}{lll}
b_{\omega}(z)=\partial_{\omega^{\prime}} a_{\omega \omega^{\prime}}(z) & \text { i.e } & b(z)=\nabla a(z) \\
c(z)=\partial_{\omega \omega^{\prime}} a_{\omega \omega^{\prime}}(z) & \text { i.e } & c(z)=\nabla^{2} a(z)
\end{array}\right.
$$

and without possible confusion, adopt the following notations,

$$
\bar{a}=a * f, \bar{b}=b * f \text { and } \bar{c}=c * f .
$$

Integrating (1.2) by parts, we can then write (1.1) alternatively under the form

$$
\partial_{t} f=\bar{a}_{\omega \omega^{\prime}} \partial_{\omega \omega^{\prime}} f-\bar{c} f \quad \text { i.e } \quad \partial_{t} f=\bar{a} \nabla^{2} f-\bar{c} f .
$$

Notations: For all $s \geq 0$, we shall use the weighted spaces $L_{s}^{1}$ and their standard norms

$$
\|f\|_{L_{s}^{1}}=\int_{\mathbb{R}^{N}}|f(v)|\left(1+|v|^{2}\right)^{\frac{s}{2}} d v .
$$

Let us recall that $\bigcap_{h \geq 0, s \geq 0} H_{s}^{h}\left(\mathbb{R}^{N}\right)$ is Schwartz's space $\mathcal{S}\left(\mathbb{R}^{N}\right)$.

Moreover, we set

$$
\begin{gathered}
M_{0}=\int_{\mathbb{R}^{N}} f_{0}(v) d v, \quad E_{0}=\frac{1}{2} \int_{\mathbb{R}^{N}} f_{0}(v)|v|^{2} d v, \\
H_{0}=\int_{\mathbb{R}^{N}} f_{0}(v) \log f_{0}(v) d v,
\end{gathered}
$$


for the initial mass, energy and entropy.

Finally, the usual entropic space is

$$
L \log L\left(\mathbb{R}^{N}\right)=\left\{f \in L^{1}\left(\mathbb{R}^{N}\right) ; \int_{\mathbb{R}^{N}} \mid f(v) \| \log (|f(v)|) d v<+\infty\right\} .
$$

Our main result is then given by

Theorem 1.1 Let $f_{0} \in L_{2+\delta}^{1} \cap \operatorname{Llog} L\left(\mathbb{R}^{N}\right)$, for some $\delta>0$. Let $f$ be any weak non negative solution of Landau homogeneous equation (1.1), (1.2) satisfying assumptions (1.4) or (1.5) and (1.7). Then, $f$ lies in $C^{\infty}\left(\left[t_{0},+\infty\right] ; \mathcal{S}\left(\mathbb{R}^{N}\right)\right)$ for all $t_{0}>0$.

The paper is organized as follows. Section 2 gives the basic tools needed to apply LittlewoodPaley theory. Section 3 is devoted to the proof of Theorem 1.1, divided in a few steps, and in the case of smooth kernels, that is under assumption (1.4). In section 4, we modify slightly the method of proof and consider the non smooth case, that is under assumption (1.5), thus concluding the paper. Finally, an appendix is devoted to an estimation of a linear operator which is crucial during the proof.

\section{Basic elements of Littlewood Paley decomposition.}

This section is devoted to Littlewood-Paley decomposition and some links with Sobolev type spaces. We use the same decomposition as in the paper of Alexandre and El Safadi [3]. More details are available in the books of Runst, Sickel, Stein and Triebel $[17,20,21]$ and also in the lecture notes by Tao [19].

We fix once for all in this paper a collection $\left\{\psi_{k}=\psi_{k}(\xi)\right\}_{k \in \mathbb{N}}$ of smooth functions such that

$$
\begin{gathered}
\operatorname{supp} \psi_{0} \subset\left\{\xi \in \mathbb{R}^{N},|\xi| \leq 2\right\}, \\
\operatorname{supp} \psi_{k} \subset\left\{\xi \in \mathbb{R}^{N}, 2^{k-1} \leq|\xi| \leq 2^{k+1}\right\} \text { for all } k \geq 1,
\end{gathered}
$$

and

$$
\sum_{k=0}^{+\infty} \psi_{k}(\xi)=1 \text { for all } \xi \in \mathbb{R}^{N} .
$$

To simplify some computations, all functions $\psi_{k}$, for $k \geq 1$, are constructed from a single one $\psi \geq 0$, i.e. we are given $\psi$ such that $\operatorname{supp} \psi \subset\left\{\xi \in \mathbb{R}^{N}, \frac{1}{2} \leq|\xi| \leq 2\right\}, \psi>0$ if 
$\frac{1}{\sqrt{2}} \leq|\xi| \leq \sqrt{2}$ such that $\psi_{k}(\xi) \equiv \psi\left(\frac{\xi}{2^{k}}\right)$, for all $k \geq 1$ and $\xi \in \mathbb{R}^{N}$.

Then, we define the real Littlewood-Paley projection operators $p_{k}$, for $k \geq 0$, by

$$
\widehat{p_{k} f}(\xi)=\psi_{k}(\xi) \hat{f}(\xi) \text {. }
$$

It follows that one has the Littlewood-Paley decomposition

$$
f=\sum_{k=0}^{+\infty} p_{k} f \text { for all } f \in \mathcal{S}^{\prime} .
$$

By construction, we can find a new collection $\left\{\tilde{\psi}_{k}=\tilde{\psi}_{k}(\xi)\right\}_{k \in \mathbb{N}}$ of smooth functions such that $\operatorname{supp} \tilde{\psi}_{0} \subset\left\{\xi \in \mathbb{R}^{N},|\xi| \leq 4\right\}, \quad \operatorname{supp} \tilde{\psi}_{k} \subset\left\{\xi \in \mathbb{R}^{N}, 2^{k-2} \leq|\xi| \leq 2^{k+2}\right\}$ for all $k \geq 1$, and such that $\psi_{k} \tilde{\psi}_{k}=\psi_{k}$, for all integer $k$.

As before, we define the corresponding operator $\tilde{p}_{k}$, for $k \geq 0$, by

$$
\widehat{\tilde{p}_{k} f}(\xi)=\tilde{\psi}_{k}(\xi) \hat{f}(\xi) \text {. }
$$

All these functions $\tilde{\psi}_{k}$, for $k \geq 1$, are constructed from a single one $\tilde{\psi} \geq 0$, i.e. we are given $\tilde{\psi}$ such that $\operatorname{supp} \tilde{\psi} \subset\left\{\xi \in \mathbb{R}^{N}, \frac{1}{2^{2}} \leq|\xi| \leq 2^{2}\right\}, \tilde{\psi}>0$ if $\frac{1}{2} \leq|\xi| \leq 2$, such that $\tilde{\psi}_{k}(\xi) \equiv \tilde{\psi}\left(\frac{\xi}{2^{k}}\right)$, for all $k \geq 1$ and $\xi \in \mathbb{R}^{N}$.

Thus, we have the obvious property, for any integer $k$

$$
p_{k} \tilde{p}_{k}=p_{k}
$$

Moreover, using Plancherel formula, these operators have the special property

$$
\int_{v} f(v) p_{k}\left(\operatorname{resp} . \tilde{p}_{k}\right) g(v) d v=\int_{v} p_{k}\left(\operatorname{resp} . \tilde{p}_{k}\right) f(v) g(v) d v, \text { for all } f, g \in \mathcal{S}^{\prime} .
$$

Using these operators, we obviously get the following Bernstein inequality, for all $f \in L^{1}$

$$
\left\|p_{k} f\right\|_{L^{2}} \leq C 2^{\frac{N k}{2}}\left\|p_{k} f\right\|_{L^{1}}
$$

where $C$ is a constant depending on the function $\tilde{\psi}$ (for the proof and more details, see proposition 3.2 on p.24 of Ref.[13]).

Thanks to this decomposition, we can see that the weighted space $L_{s}^{1}$ satisfies

$$
\forall s>0, \quad\|f\|_{L_{s}^{1}} \sim \sum_{j=0}^{\infty} 2^{j s}\left\|\psi_{j} f\right\|_{L^{1}} .
$$

Finally, usual weighted Sobolev spaces can be described by the following important result, see for instance the results quoted in the books $[17,20,21]$ 
Lemma 2.1 For all $s, j \geq 0$,

$$
\|f\|_{H_{s}^{h}}^{2} \sim \sum_{k=0}^{+\infty} \sum_{j=0}^{+\infty} 2^{k h} 2^{j s}\left\|p_{k}\left(\psi_{j} f\right)\right\|_{L^{2}}^{2}
$$

\section{Proof of Theorem 1.1, under assumption (1.4)}

It will be divided into six parts. In the first one, we make some manipulations to simplify our task and obtain a simple equality to estimate. Then, in the next three parts, we estimate each term appearing in this equality, and glue all these estimates in the fifth part, getting a differential inequality. Finally, in the last one, we deduce an estimation of our solution in Schwartz's space w.r.t. variable $v$ and $C^{\infty}$ w.r.t. time $t$.

\subsection{Simplification step}

We shall use the Landau equation under the form given by (1.9)

$$
\partial_{t} f=\bar{a} \nabla^{2} f-\bar{c} f
$$

Next, let $k$ and $j$ be any positive integers. We make the convention that all functions $\psi_{j}$ appearing with negative indices, are equal to zero (in fact we need only $\psi_{-2}, \psi_{-1}$ ).

Multiplying (3.13) by $\psi_{j}$, thanks to the equality $\tilde{\psi}_{j} \psi_{j}=\psi_{j}$, we get

$$
\partial_{t} \psi_{j} f=\left(\bar{a} \tilde{\psi}_{j}\right) \psi_{j} \nabla^{2} f-\left(\bar{c} \tilde{\psi}_{j}\right) \psi_{j} f
$$

Since supp $\nabla \psi_{j} \subset \operatorname{supp} \psi_{j}$, we get

$\psi_{j} \nabla^{2} f=\nabla^{2}\left(\psi_{j} f\right)-\sum_{m=j-2}^{j+2}\left(\nabla^{2} \psi_{j}\right) \psi_{m} f-2 \sum_{m=j-2}^{j+2}\left(\nabla \psi_{j}\right) \nabla\left(\psi_{m} f\right)+\sum_{m=j-2}^{j+2} \sum_{n=j-2}^{j+2}\left(\nabla \psi_{j}\right)\left(\nabla \psi_{m}\right) \psi_{n} f$.

Taking into account $(3.15)$ and the fact $\left(\nabla \psi_{j}\right) \tilde{\psi}_{j}=\nabla \psi_{j}$, equality (3.14) becomes

$$
\begin{aligned}
\partial_{t} \psi_{j} f & =\left(\bar{a} \tilde{\psi}_{j}\right) \nabla^{2}\left(\psi_{j} f\right)-\left(\bar{c} \tilde{\psi}_{j}\right) \psi_{j} f-\sum_{m=j-2}^{j+2} \bar{a}\left(\nabla^{2} \psi_{j}\right) \psi_{m} f \\
& -2 \sum_{m=j-2}^{j+2} \bar{a}\left(\nabla \psi_{j}\right) \nabla\left(\psi_{m} f\right) \sum_{m=j-2}^{j+2}+\sum_{n=j-2}^{j+2} \bar{a}\left(\nabla \psi_{j}\right)\left(\nabla \psi_{m}\right) \psi_{n} f .
\end{aligned}
$$


Hereafter, for simplicity, we shall use variables $m, n$ to notify a finite summation on $m$ and $n$. More precisely, variable $m$ (resp. $n$ ) denotes summation on variable $m$ ( $\operatorname{resp} . n$ ) ranging from $j-2$ to $j+2$.

Applying Littlewood Paley operator $p_{k}$ to (3.16), we get

$$
\left\{\begin{array}{l}
\partial_{t} p_{k}\left(\psi_{j} f\right)=\left[p_{k}, \bar{a} \tilde{\psi}_{j}\right] \nabla^{2}\left(\psi_{j} f\right)+\left(\bar{a} \tilde{\psi}_{j}\right) \nabla^{2} p_{k}\left(\psi_{j} f\right)-\left[p_{k}, \bar{c} \tilde{\psi}_{j}\right] \psi_{j} f-\left(\bar{c} \tilde{\psi}_{j}\right) p_{k}\left(\psi_{j} f\right) \\
-\left[p_{k}, \bar{a} \nabla^{2}\left(\psi_{j}\right)\right] \psi_{m} f-\bar{a} \nabla^{2}\left(\psi_{j}\right) p_{k}\left(\psi_{m} f\right)-2\left[p_{k}, \bar{a}\left(\nabla \psi_{j}\right)\right] \nabla\left(\psi_{m} f\right)-2 \bar{a}\left(\nabla \psi_{j}\right) \nabla p_{k}\left(\psi_{m} f\right) \\
+\left[p_{k}, \bar{a}\left(\nabla \psi_{j}\right)\left(\nabla \psi_{m}\right)\right] \psi_{n} f+\bar{a}\left(\nabla \psi_{j}\right)\left(\nabla \psi_{m}\right) p_{k}\left(\psi_{n} f\right) .
\end{array}\right.
$$

After some computations, using integrations by parts, we get

$$
\begin{array}{r}
{\left[p_{k}, \bar{a} \tilde{\psi}_{j}\right] \nabla^{2}\left(\psi_{j} f\right)=\left[\nabla^{2} p_{k}, \bar{a} \tilde{\psi}_{j}\right] \psi_{j} f-2\left[\nabla p_{k}, \nabla\left(\bar{a} \tilde{\psi}_{j}\right)\right] \psi_{j} f-2 \nabla\left(\bar{a} \tilde{\psi}_{j}\right) \nabla p_{k}\left(\psi_{j} f\right)} \\
+\left[p_{k}, \bar{a} \nabla^{2}\left(\tilde{\psi}_{j}\right)\right] \psi_{j} f+2\left[p_{k}, \bar{b} \nabla\left(\tilde{\psi}_{j}\right)\right] \psi_{j} f+\left[p_{k}, \bar{c} \tilde{\psi}_{j}\right] \psi_{j} f \\
+\bar{a} \nabla^{2}\left(\tilde{\psi}_{j}\right) p_{k}\left(\psi_{j} f\right)+2 \bar{b} \nabla\left(\tilde{\psi}_{j}\right) p_{k}\left(\psi_{j} f\right)+\bar{c} \tilde{\psi}_{j} p_{k}\left(\psi_{j} f\right)
\end{array}
$$

and

$$
\begin{array}{r}
{\left[p_{k}, \bar{a}\left(\nabla \psi_{j}\right)\right] \nabla\left(\psi_{m} f\right)=\left[\nabla p_{k}, \bar{a} \nabla \psi_{j}\right] \psi_{m} f-\left[p_{k}, \bar{b} \nabla\left(\psi_{j}\right)\right] \psi_{m} f-\left[p_{k}, \bar{a} \nabla^{2}\left(\psi_{j}\right)\right] \psi_{m} f} \\
-\bar{b} \nabla\left(\psi_{j}\right) p_{k}\left(\psi_{m} f\right)-\bar{a} \nabla^{2}\left(\psi_{j}\right) p_{k}\left(\psi_{m} f\right) .
\end{array}
$$

Plugging (3.18) and (3.19) into equality (3.17), multiplying the result by $p_{k}\left(\psi_{j} f\right)$ and integrating w.r.t. variable $v$, we find the following equality (recall that $m$ and $n$ denotes in fact a double summation $\sum_{m} \sum_{n}$ )

$$
\begin{gathered}
\partial_{t}\left\|p_{k}\left(\psi_{j} f\right)\right\|_{L^{2}}^{2}-\frac{1}{2} \int_{v} \bar{a} \tilde{\psi}_{j} \nabla^{2} p_{k}\left(\psi_{j} f\right) p_{k}\left(\psi_{j} f\right) d v \\
=\frac{1}{2} \int_{v}\left[\nabla^{2} p_{k}, \bar{a} \tilde{\psi}_{j}\right]\left(\psi_{j} f\right) p_{k}\left(\psi_{j} f\right) d v \\
-\int_{v}\left[\nabla p_{k}, \nabla\left(\bar{a} \tilde{\psi}_{j}\right)\right]\left(\psi_{j} f\right) p_{k}\left(\psi_{j} f\right) d v-\int_{v} \nabla\left(\bar{a} \tilde{\psi}_{j}\right) \nabla p_{k}\left(\psi_{j} f\right) p_{k}\left(\psi_{j} f\right) d v \\
+\frac{1}{2} \int_{v}\left[p_{k}, \bar{a} \nabla^{2} \tilde{\psi}_{j}+2 \bar{b} \nabla \tilde{\psi}_{j}\right]\left(\psi_{j} f\right) p_{k}\left(\psi_{j} f\right) d v+\frac{1}{2} \int_{v}\left(\bar{a} \nabla^{2} \tilde{\psi}_{j}+2 \bar{b} \nabla \tilde{\psi}_{j}\right) p_{k}\left(\psi_{j} f\right) p_{k}\left(\psi_{j} f\right) d v \\
+\frac{1}{2} \int_{v}\left[p_{k}, \bar{a} \nabla^{2} \psi_{j}+2 \bar{b} \nabla \psi_{j}\right]\left(\psi_{m} f\right) p_{k}\left(\psi_{j} f\right) d v+\frac{1}{2} \int_{v}\left(\bar{a} \nabla^{2} \psi_{j}+2 \bar{b} \nabla \psi_{j}\right) p_{k}\left(\psi_{m} f\right) p_{k}\left(\psi_{j} f\right) d v \\
+\frac{1}{2} \int_{v}\left[p_{k}, \bar{a} \nabla \psi_{j}, \bar{a} \nabla \psi_{j}\right]\left(\psi_{m} f\right) p_{k}\left(\psi_{j} f\right) d v-\int_{n} \bar{a} \nabla \psi_{j} \nabla p_{k}\left(\psi_{m} f\right) p_{k}\left(\psi_{j} f\right) d v
\end{gathered}
$$


Hereafter, $C$ denotes any positive constant not depending on the integers $k$ or $j$ that will appear in the proof of our results below. If we need to mention its dependence with respect to any parameter $\beta$, then we shall use the notation $C_{\beta}$. Again, let us recall that variables $m$ and $n$ appearing in this work, are a shorthand notation to signify a summation on these variables between the two integers $j-2$ and $j+2$.

\subsection{Lower bounds}

Firstly, integrating by parts, the second term of (3.20) becomes

$$
\begin{aligned}
-\frac{1}{2} \int_{v}\left(\bar{a} \tilde{\psi}_{j}\right) \nabla^{2} p_{k}\left(\psi_{j} f\right) p_{k}\left(\psi_{j} f\right) d v & = \\
\frac{1}{2} \int_{v} \nabla\left(\bar{a} \tilde{\psi}_{j}\right) \nabla p_{k}\left(\psi_{j} f\right) p_{k}\left(\psi_{j} f\right) d v & +\frac{1}{2} \int_{v}\left(\bar{a} \tilde{\psi}_{j}\right) \nabla p_{k}\left(\psi_{j} f\right) \nabla p_{k}\left(\psi_{j} f\right) d v .
\end{aligned}
$$

We want to estimate from below the second term on the right hand side of (3.27), and here we shall use the ellipticity of the matrix $\bar{a}_{\omega \omega^{\prime}}$, see [9] (proposition 4). The degeneracy of the matrix $\Pi_{\omega \omega^{\prime}}$ entails a loss of order 2 in the exponent w.r.t. $|v|$. However, their proof, which is given for $\mathcal{X}(|v|)=|v|^{\gamma}$, holds true also for $\mathcal{X}(|v|)=\left(1+|v|^{2}\right)^{\frac{\gamma}{2}}$.

Thus, one has

$$
\bar{a}_{\omega \omega^{\prime}}(v) \xi_{\omega} \xi_{\omega^{\prime}} \geq K\left(1+|v|^{2}\right)^{\frac{\gamma}{2}}|\xi|^{2} \quad \forall \xi \in \mathbb{R}^{N}
$$

where $K$ is a non negative constant depending only on the initial moment $M_{0}$, energy $E_{0}$, entropy $H_{0}$ and $\gamma$. Taking into account the support of our basic functions, we obtain

$$
\bar{a} \tilde{\psi}_{j} \nabla p_{k}\left(\psi_{j} f\right) \nabla p_{k}\left(\psi_{j} f\right) \geq K \mathcal{C}_{j}^{\frac{\gamma}{2}} \tilde{\psi}_{j} \nabla p_{k}\left(\psi_{j} f\right) \nabla p_{k}\left(\psi_{j} f\right) \quad \text { where } \mathcal{C}_{j}=\left(1+2^{2 j}\right)
$$

But, using the fact, $\tilde{\psi}_{j} \psi_{j}=\psi_{j}$, we get

$$
\tilde{\psi}_{j} \nabla p_{k} \psi_{j} f=\nabla\left(p_{k} \psi_{j} f\right)-\left[\nabla p_{k}, \tilde{\psi}_{j}\right] \psi_{j} f
$$

Then, after some computations, integrating by parts, we get

$$
\begin{aligned}
\frac{1}{2} \int_{v} \bar{a} \tilde{\psi}_{j} \nabla p_{k}\left(\psi_{j} f\right) \nabla p_{k}\left(\psi_{j} f\right) d v & \geq K \mathcal{C}_{j}^{\frac{\gamma}{2}} \int_{v} \nabla p_{k}\left(\psi_{j} f\right) \nabla p_{k}\left(\psi_{j} f\right) d v \\
+K \mathcal{C}_{j}^{\frac{\gamma}{2}} \int_{v}\left[\nabla^{2} p_{k}, \tilde{\psi}_{j}\right] \psi_{j} f p_{k}\left(\psi_{j} f\right) d v & -K \mathcal{C}_{j}^{\frac{\gamma}{2}} \int_{v}\left(\nabla \tilde{\psi}_{j}\right) \nabla p_{k}\left(\psi_{j} f\right) p_{k}\left(\psi_{j} f\right) d v .
\end{aligned}
$$

As a consequence of this section, we have obtained a term bounded from below (that is, the second integral of (3.28)) which will be written on the left-hand side of the last equality, while for all the other terms, adding the two integrals of (3.29) and the first integral of (3.27), we shall find an upper bound for each one to finally obtain a differential inequality. 


\subsection{Estimates: Upper bounds}

We divide this section into three parts. In the first one, we give an estimation of the matrix $\bar{a}$, defined in (1.3), (1.8) and its derivatives, which will be useful in the next parts. Then, in the second, we introduce two lemmas giving upper bounds which will be useful to implement an iteration step and finally, we estimate the remaining terms of the equality from section 2 .

\subsubsection{Estimates for $\bar{a}$ and $\bar{b}$.}

Firstly, let us note that under assumption (1.4), since $\chi$ is taken very smooth, it follows that $a$ belongs to $C^{\infty}\left(\mathbb{R}^{N}\right)$. Taking into account notations (1.8), one has, for all $v \in \mathbb{R}^{N}$

$$
\begin{gathered}
b(v)=(1-N) v \chi(|v|), \\
\nabla^{\beta} a(v)=(1-N)\left[(N+\beta-2) \nabla^{\beta-2}(\chi(|v|))+v \nabla^{\beta-1}(\chi(|v|))\right], \text { for all integer } \beta \geq 2 .
\end{gathered}
$$

In view of the convolution structure, using the following inequality

$$
\forall v, v_{*} \in \mathbb{R}^{N} \quad\left(1+\left|v-v_{*}\right|^{2}\right)^{\frac{\gamma}{2}} \leq\left(1+|v|^{2}\right)^{\frac{\gamma}{2}}\left(1+\left|v_{*}\right|^{2}\right)^{\frac{\gamma}{2}},
$$

we get

$$
\begin{gathered}
|\bar{a}(v)| \leq C\left(1+|v|^{2}\right)^{\frac{\gamma+2}{2}}, \quad|\bar{b}(v)| \leq C\left(1+|v|^{2}\right)^{\frac{\gamma+1}{2}}, \\
\left|\nabla^{\beta}(\bar{a}(v))\right| \leq C C_{\beta}\left(1+|v|^{2}\right)^{\frac{\gamma}{2}} \quad \forall \beta \geq 2,
\end{gathered}
$$

for a constant $\mathrm{C}$ depending here only on $\|f\|_{L_{\gamma+2}^{1}}$.

Therefore, we obtain, using the precise support sets of $\psi_{j}$ and $\tilde{\psi}_{j}$, the following inequalities, for all $j \in \mathbb{N}$

$$
\left\{\begin{array}{l}
\left|\bar{a} \psi_{j}\right| \leq C \mathcal{C}_{j}^{\frac{\gamma+2}{2}},\left|\bar{a} \nabla \psi_{j}\right| \leq C \mathcal{C}_{j}^{\frac{\gamma+1}{2}},\left|\bar{a} \nabla^{2} \psi_{j}\right| \leq C \mathcal{C}_{j}^{\frac{\gamma}{2}}, \\
\left|\bar{b} \psi_{j}\right| \leq C \mathcal{C}_{j}^{\frac{\gamma+1}{2}},\left|\bar{b} \nabla \psi_{j}\right| \leq C \mathcal{C}_{j}^{\frac{\gamma}{2}} \\
\text { and }\left|\nabla^{\beta}\left(\bar{a} \psi_{j}\right)\right| \leq C C_{\beta} \mathcal{C}_{j}^{\frac{\gamma}{2}}, \quad \forall \beta \geq 2,
\end{array}\right.
$$

for a constant $\mathrm{C}$ depending on $\|f\|_{L_{\gamma+2}^{1}}$ and the bound $L^{\infty}$ of $\psi$ and their derivatives. Of course, these inequalities also hold true if we change $\psi_{j}$ by $\tilde{\psi}_{j}$.

\subsubsection{Integral operators estimates}

This subsection is concerned with a linear operator having the form $\left[\nabla^{r} p_{k}, \phi\right]$, for orders $r=0,1,2$, where $p_{k}$ is the Littlewood-Paley operator (see section 2) and $\phi$ is any smooth function. We will introduce two lemmas, which will play an essential role in order to obtain the needed upper bounds. 
Before that, let us do some computations concerning the operator $\tilde{p}_{k}$ which will be useful in the sequel for our bounds into the lemmas.

According to section 2, we know that $\widehat{\tilde{p}_{k} g}$ is supported in $2^{k-2} \leq|\xi| \leq 2^{k+2}$, for any function $g \in L^{1}$. This implies, by the Littlewood decomposition, that

$$
\left.\widehat{\tilde{p}_{k} g}=\widehat{p_{k-3}\left(\tilde{p}_{k}\right.} g\right)+\widehat{p_{k-2}\left(\tilde{p}_{k} g\right)}+\widehat{p_{k-1}\left(\tilde{p}_{k} g\right)}+\widehat{p_{k}\left(\tilde{p}_{k} g\right)}+\widehat{p_{k+1}\left(\tilde{p}_{k} g\right)}+\widehat{p_{k+2}\left(\tilde{p}_{k} g\right)}+\widehat{p_{k+3}\left(\tilde{p}_{k} g\right)} \text {. }
$$

Again, all operators indexed by negative indices are set to zero (that is here $p_{-3}, p_{-2}, p_{-1}$ ). Thanks to the commutativity property of operator $p_{k}$, we have

$$
\widehat{\tilde{p}_{k} g}=\tilde{p}_{k} \widehat{\left(p_{k-3} g\right)}+\tilde{p}_{k} \widehat{\left(p_{k-2} g\right)}+\tilde{p}_{k} \widehat{\left(p_{k-1} g\right)}+\widehat{\tilde{p}_{k}\left(p_{k} g\right)}+\tilde{p}_{k} \widehat{\left(p_{k+1} g\right)}+\tilde{p}_{k} \widehat{\left(p_{k+2} g\right)}+\tilde{p}_{k} \widehat{\left(p_{k+3} g\right)} .
$$

Hence, by Parseval's inequality, we get

$$
\begin{aligned}
\left\|\tilde{p}_{k} g\right\|_{L^{2}} & \leq \sum_{l=k-3}^{k+3}\left\|\tilde{p}_{k}\left(p_{l} g\right)\right\|_{L^{2}} \\
& \leq C \sum_{l=k-3}^{k+3}\left\|p_{l} g\right\|_{L^{2}}
\end{aligned}
$$

where $C=\|\tilde{\psi}\|_{L^{\infty}}$.

We begin with the following estimate:

Lemma 3.1 Let $r \in \mathbb{N}^{*}$ and $k \in \mathbb{N}$. Let $\phi$ be a bounded continuous function together with its derivatives. Then, for a function $f \in L^{1}$, we have the following estimate,

$\left|\int_{v}\left[\nabla^{2} p_{k}, \phi\right] f p_{k} f d v\right| \leq \frac{C\left\|\nabla^{r+1} \phi\right\|_{L^{\infty}}}{2^{k(r-1)}}\|f\|_{L^{1}} 2^{\frac{N k}{2}}\left\|p_{k} f\right\|_{L^{2}}+\beta_{r} \sum_{l=k-3}^{k+3}\left\|p_{l} f\right\|_{L^{2}}\left\|\nabla p_{k} f\right\|_{L^{2}}$

where

$$
\beta_{r}=C\left\{\left\|\nabla^{1} \phi\right\|_{L^{\infty}}+\left\|\nabla^{2} \phi\right\|_{L^{\infty}}+\cdots+\left\|\nabla^{r} \phi\right\|_{L^{\infty}}\right\}
$$

and $C$ is a constant not depending on the variable $k$.

Proof: We shall use iterations provided by the adjoint property $(2.11)$ of $p_{k}$, and apply it several times. This method allows us to get negative exponents of $k$ which play an essential role to obtain our regularity. After $r$ iterations, this term writes

$$
\int_{v}\left[\nabla^{2} p_{k}, \phi\right] f p_{k} f d v=\int_{v} \tilde{T}_{k}^{r, 2}(f) p_{k} f d v+\sum_{m=0}^{r-1} \int_{v} \tilde{T}_{k}^{m, 2}\left(\tilde{p}_{k} f\right) p_{k} f d v
$$


where

$$
\tilde{T}_{k}^{\tau, 2}=\overbrace{\left[\tilde{p}_{k},\left[\tilde{p}_{k},\left[\tilde{p}_{k}, \cdots\right.\right.\right.}^{\tau},\left[\nabla^{2} p_{k}, \phi\right]]]] \quad \text { for all } \tau \in \mathbb{N} .
$$

Using the result quoted in the Appendix, this linear operator has an $L^{p}$ estimate, for $1 \leq$ $p \leq \infty$, as follows

$$
\left\|\tilde{T}_{k}^{\tau, 2}\right\|_{L^{p} \rightarrow L^{p}} \leq \frac{C_{\tau}^{\prime}}{2^{k(l-1)}}, \text { with } C_{\tau}^{\prime}=C\left\|\nabla^{\tau+1} \varphi\right\|_{L^{\infty}}
$$

As regards the first term of the right hand side of (3.59), we have

$$
\begin{aligned}
\left|\int_{v} \tilde{T}_{k}^{r, 2}(f) p_{k} f d v\right| & \leq\left\|\tilde{T}_{k}^{r, 2}(f)\right\|_{L^{1}}\left\|p_{k} f\right\|_{L^{\infty}} \quad \text { (Holder inequality) } \\
& \leq\left\|\tilde{T}_{k}^{r, 2}(f)\right\|\left\|_{L^{1}} 2^{\frac{N k}{2}}\right\| p_{k} f \|_{L^{2}} \quad \text { (Bernstein inequality 2.12) } \\
& \leq \frac{C_{r}^{\prime}}{2^{(r-1) k}}\|f\|_{L^{1}} 2^{\frac{N k}{2}}\left\|p_{k} f\right\|_{L^{2}} \text { (see 3.44). }
\end{aligned}
$$

For the second estimation, one has

$$
\begin{aligned}
\left|\sum_{m=1}^{r-1} \int_{v} \tilde{T}_{k}^{m, 2}\left(\tilde{p}_{k} f\right) p_{k} f d v\right| & \leq \sum_{m=1}^{r-1}\left\|\tilde{T}_{k}^{m, 2}\left(\tilde{p}_{k} f\right)\right\|_{L^{2}}\left\|p_{k} f\right\|_{L^{2}} \quad \text { (Cauchy Schwarz inequality) } \\
& \leq \beta_{r}^{\prime} 2^{k}\left\|\tilde{p}_{k} f\right\|_{L^{2}}\left\|p_{k} f\right\|_{L^{2}} \text { (see 3.44) }
\end{aligned}
$$

where $\beta_{r}^{\prime}=C \sum_{m=0}^{r-1}\left\|\nabla^{m+1} \phi\right\|_{L^{\infty}}$.

Taking into account the inequality $\left\|\nabla p_{k} f\right\|_{L^{2}} \geq C 2^{k}\left\|p_{k} f\right\|_{L^{2}}$ obtained by Plancherel formula, using (3.31), the last term (3.35) has the following upper bound

$$
\beta_{r}^{\prime} \sum_{l=k-3}^{k+3}\left\|p_{l} f\right\|_{L^{2}}\left\|\nabla p_{k} f\right\|_{L^{2}} .
$$

Thanks to (3.34) and (3.36), we get the lemma.

Next, let us take any integer $r$ such that $r>0$. Its value will be choosen exactly later. Applying Lemma 3.1 to the first integral of (3.29), we obtain

$$
\begin{aligned}
K \mathcal{C}_{j}^{\frac{\gamma}{2}}\left|\int_{v}\left[\nabla^{2} p_{k}, \tilde{\psi}_{j}\right] \psi_{j} f p_{k}\left(\psi_{j} f\right) d v\right| & \leq \frac{C_{r} \mathcal{C}_{j}^{\frac{\gamma}{2}}}{2^{k(r-1)}}\left\|\psi_{j} f\right\|_{L^{1}} 2^{\frac{N k}{2}}\left\|p_{k}\left(\psi_{j} f\right)\right\|_{L^{2}} \\
& +C_{r} \mathcal{C}_{j}^{\frac{\gamma}{2}} \sum_{l=k-3}^{k+3}\left\|p_{l}\left(\psi_{j} f\right)\right\| L_{L^{2}}\left\|\nabla p_{k}\left(\psi_{j} f\right)\right\|_{L^{2}}
\end{aligned}
$$


Here, $C_{r}$ is a constant depending only on $K,\|\tilde{\psi}\|_{L^{\infty}}$ and on variable $r$.

For any constant $\varepsilon>0$, by Young's inequality, each term of (3.38) writes, for $l=k-3, k-$ $2, k-1, k, k+1, k+2, k+3$

$$
\frac{C_{r} \mathcal{C}_{j}^{\frac{\gamma}{2}}}{\varepsilon}\left\|p_{l}\left(\psi_{j} f\right)\right\|_{L^{2}} \varepsilon\left\|\nabla p_{k}\left(\psi_{j} f\right)\right\|_{L^{2}} \leq \frac{C_{r} \mathcal{C}_{j}^{\gamma}}{2 \varepsilon^{2}}\left\|p_{l}\left(\psi_{j} f\right)\right\|_{L^{2}}^{2}+\frac{\varepsilon^{2}}{2}\left\|\nabla p_{k}\left(\psi_{j} f\right)\right\|_{L^{2}}^{2} .
$$

Concerning the integral (3.21), using Lemma 3.1 and (3.30), we obtain

$$
\begin{aligned}
\frac{1}{2}\left|\int_{v}\left[\nabla^{2} p_{k}, \bar{a} \tilde{\psi}_{j}\right]\left(\psi_{j} f\right) p_{k}\left(\psi_{j} f\right) d v\right| & \leq \frac{C_{r} \mathcal{C}_{j}^{\frac{\gamma}{2}}}{2^{k(r-1)}}\left\|\psi_{j} f\right\|_{L^{1}} 2^{\frac{N k}{2}}\left\|p_{k}\left(\psi_{j} f\right)\right\|_{L^{2}} \\
& +C_{r} \mathcal{C}_{j}^{\frac{\gamma+1}{2}} \sum_{l=k-3}^{k+3}\left\|p_{l}\left(\psi_{j} f\right)\right\| L_{L^{2}}\left\|\nabla p_{k}\left(\psi_{j} f\right)\right\|_{L^{2}}(3.4
\end{aligned}
$$

Again, as for (3.39), for any constant $\eta>0$, thanks to Young's inequality, the term (3.41) has the following upper bound

$$
\frac{C_{r} \mathcal{C}_{j}^{\frac{\gamma+1}{2}}}{\eta}\left\|p_{l}\left(\psi_{j} f\right)\right\|_{L^{2}} \eta\left\|\nabla p_{k}\left(\psi_{j} f\right)\right\|_{L^{2}} \leq \frac{C_{r} \mathcal{C}_{j}^{\gamma+1}}{2 \eta^{2}}\left\|p_{l}\left(\psi_{j} f\right)\right\|_{L^{2}}^{2}+\frac{\eta^{2}}{2}\left\|\nabla p_{k}\left(\psi_{j} f\right)\right\|_{L^{2}}^{2} .
$$

The following Lemma is the counterpart of the previous one, but for lower order derivatives.

Lemma 3.2 Let $r \in \mathbb{N}^{*}, k \in \mathbb{N}$ and $h=0,1$. Let $\phi$ a bounded continuous function together with its derivatives. Then, for a function $f \in L^{1}$, we have the following estimate

$\left|\int_{v}\left[\nabla^{h} p_{k}, \phi\right] f p_{k} f d v\right| \leq \frac{C\left\|\nabla^{r+1} \phi\right\|_{L^{\infty}}}{2^{k(r+1-h)}}\|f\|_{L^{1}} 2^{\frac{N k}{2}}\left\|p_{k} f\right\|_{L^{2}}+\beta_{r}\left[\sum_{l=k-3}^{k+3}\left\|p_{l} f\right\|_{L^{2}}^{2}+\left\|p_{k} f\right\|_{L^{2}}^{2}\right]$,

where

$$
\beta_{r}=C\left\{\left\|\nabla^{1} \phi\right\|_{L^{\infty}}+\left\|\nabla^{2} \phi\right\|_{L^{\infty}}+\cdots+\left\|\nabla^{r} \phi\right\|_{L^{\infty}}\right\}
$$

and $C$ is a constant not depending on the variable $k$.

Proof: Taking into account property (2.11), we can write, after $r$ iterations

$$
\int_{v}\left[\nabla^{h} p_{k}, \phi\right] f p_{k} f d v=\int_{v} \tilde{T}_{k}^{r, h}(f) p_{k} f d v+\sum_{m=0}^{r-1} \int_{v} \tilde{T}_{k}^{m, h}\left(\tilde{p}_{k} f\right) p_{k} f d v
$$

where

$$
\tilde{T}_{k}^{\tau, h}=\overbrace{\left[\tilde{p}_{k},\left[\tilde{p}_{k},\left[\tilde{p}_{k}, \cdots\right.\right.\right.}^{\tau},\left[\nabla^{h} p_{k}, \phi\right]]]] \quad \text { for all } \tau \in \mathbb{N} .
$$


which has the following norm estimation, for $1 \leq p \leq \infty$ (see appendix)

$$
\left\|\tilde{T}_{k}^{\tau, h}\right\|_{L^{p} \rightarrow L^{p}} \leq \frac{C_{\tau}^{\prime}}{2^{(\tau+1-h) k}} \text { with } C_{\tau}^{\prime}=C\left\|\nabla^{\tau+1} \phi\right\|_{L^{\infty}} .
$$

Concerning the first term on the left hand side of (3.43), we have

$$
\begin{aligned}
\left|\int_{v} \tilde{T}_{k}^{r, h}(f) p_{k} f d v\right| & \leq\left\|\tilde{T}_{k}^{r, h}(f)\right\|_{L^{1}}\left\|p_{k} f\right\|_{L^{\infty}} \quad \text { (Holder inequality) } \\
& \leq\left\|\tilde{T}_{k}^{r, h}(f)\right\|\left\|_{L^{1}} 2^{\frac{N k}{2}}\right\| p_{k} f \|_{L^{2}} \quad \text { (Bernstein inequality 2.12) } \\
& \leq \frac{C_{r}^{\prime}}{2^{(r+1-h) k}}\|f\|_{L^{1}} 2^{\frac{N k}{2}}\left\|p_{k} f\right\|_{L^{2}} \text { (see 3.44). }
\end{aligned}
$$

For the second, we use Cauchy Schwarz inequality to get

$$
\begin{aligned}
\left|\sum_{m=0}^{r-1} \int_{v} \tilde{T}_{k}^{m, h}\left(\tilde{p}_{k} f\right) p_{k} f d v\right| & \leq \sum_{m=0}^{r-1}\left\|\tilde{T}_{k}^{m, h}\left(\tilde{p}_{k} f\right)\right\|_{L^{2}}\left\|p_{k} f\right\|_{L^{2}} \\
& \leq \beta_{r}\left\|\tilde{p}_{k} f\right\|_{L^{2}}\left\|p_{k} f\right\|_{L^{2}}(\text { see } 3.44)
\end{aligned}
$$

where $\beta_{r}=C \sum_{m=0}^{r-1}\left\|\nabla^{m+1} \phi\right\|_{L^{\infty}}$.

Using (3.31), then, applying Young's inequality to each term of the sommation of (3.46) implies the end of the proof.

Now, we return to the first integral of (3.22), and using Lemma 3.2 with $\mathrm{h}=1$, thanks to (3.30), we have

$$
\begin{gathered}
\left|\int_{v}\left[\nabla p_{k}, \nabla\left(\bar{a} \tilde{\psi}_{j}\right)\right]\left(\psi_{j} f\right) p_{k}\left(\psi_{j} f\right) d v\right| \leq \frac{C_{r} \mathcal{C}_{j}^{\frac{\gamma}{2}}}{2^{k r}}\left\|\psi_{j} f\right\|_{L^{1}} 2^{\frac{N k}{2}}\left\|p_{k}\left(\psi_{j} f\right)\right\|_{L^{2}} \\
+C_{r} C_{j}^{\frac{\gamma}{2}}\left[\sum_{l=k-3}^{k+3}\left\|p_{l}\left(\psi_{j} f\right)\right\|_{L^{2}}^{2}+\left\|p_{k}\left(\psi_{j} f\right)\right\|_{L^{2}}^{2}\right] .
\end{gathered}
$$

Here $C_{r}$ is any constant depending only on the variable $r$.

Similarly, the first integral of (3.24) can be estimated as

$$
\begin{gathered}
\left|\int_{v}\left[\nabla p_{k}, \bar{a} \nabla \psi_{j}\right]\left(\psi_{m} f\right) p_{k}\left(\psi_{j} f\right) d v\right| \leq \frac{C_{r} \mathcal{C}_{j}^{\frac{\gamma}{2}}}{2^{k r}}\left\|\psi_{m} f\right\|_{L^{1}} 2^{\frac{N k}{2}}\left\|p_{k}\left(\psi_{j} f\right)\right\|_{L^{2}} \\
+C_{r} C_{j}^{\frac{\gamma}{2}}\left[\sum_{l=k-3}^{k+3}\left\|p_{l}\left(\psi_{m} f\right)\right\|_{L^{2}}^{2}+\left\|p_{k}\left(\psi_{j} f\right)\right\|_{L^{2}}^{2}\right] .
\end{gathered}
$$

Again, using Lemma 3.2 with $h=0$ to estimate the first integral of (3.23), we obtain

$$
\frac{1}{2}\left|\int_{v}\left[p_{k}, \bar{a} \nabla^{2} \tilde{\psi}_{j}+2 \bar{b} \nabla \tilde{\psi}_{j}\right]\left(\psi_{j} f\right) p_{k}\left(\psi_{j} f\right) d v\right| \leq \frac{C_{r} \mathcal{C}_{j}^{\frac{\gamma}{2}}}{2^{k r}}\left\|\psi_{j} f\right\|_{L^{1}} 2^{\frac{N k}{2}}\left\|p_{k}\left(\psi_{j} f\right)\right\|_{L^{2}}
$$




$$
+C_{r} C_{j}^{\frac{\gamma}{2}}\left[\sum_{l=k-3}^{k+3}\left\|p_{l}\left(\psi_{j} f\right)\right\|_{L^{2}}^{2}+\left\|p_{k}\left(\psi_{j} f\right)\right\|_{L^{2}}^{2}\right] .
$$

Similarly, for the first integral of (3.25), we have

$$
\begin{aligned}
\frac{1}{2} \mid \int_{v}\left[p_{k}, \bar{a} \nabla^{2} \psi_{j}\right. & \left.+2 \bar{b} \nabla \psi_{j}\right]\left(\psi_{m} f\right) p_{k}\left(\psi_{j} f\right) d v \mid \leq \frac{C_{r} \mathcal{C}_{j}^{\frac{\gamma}{2}}}{2^{k(r+1)}}\left\|\psi_{m} f\right\|_{L^{1}} 2^{\frac{N k}{2}}\left\|p_{k}\left(\psi_{j} f\right)\right\|_{L^{2}} \\
& +C_{r} C_{j}^{\frac{\gamma}{2}}\left[\sum_{l=k-3}^{k+3}\left\|p_{l}\left(\psi_{m} f\right)\right\|_{L^{2}}^{2}+\left\|p_{k}\left(\psi_{j} f\right)\right\|_{L^{2}}^{2}\right] .
\end{aligned}
$$

Finally, using the inequalities,

$$
\left|\bar{a}\left(\nabla \psi_{j}\right)\left(\nabla \psi_{m}\right)\right| \leq C \mathcal{C}_{j}^{\frac{\gamma}{2}}, \quad\left|\nabla^{r}\left(\bar{a}\left(\nabla \psi_{j}\right)\left(\nabla \psi_{m}\right)\right)\right| \leq C C_{r} \mathcal{C}_{j}^{\frac{\gamma}{2}},
$$

applying Lemma 3.2 with $h=0$ for the first integral of (3.26), we have

$$
\begin{gathered}
\frac{1}{2}\left|\int_{v}\left[p_{k}, \bar{a}\left(\nabla \psi_{j}\right)\left(\nabla \psi_{m}\right)\right]\left(\psi_{n} f\right) p_{k}\left(\psi_{j} f\right) d v\right| \leq \frac{C_{r} \mathcal{C}_{j}^{\frac{\gamma}{2}}}{2^{k(r+1)}}\left\|\psi_{n} f\right\|_{L^{1}} 2^{\frac{N k}{2}}\left\|p_{k}\left(\psi_{j} f\right)\right\|_{L^{2}} \\
+C_{r} C_{j}^{\frac{\gamma}{2}}\left[\sum_{l=k-3}^{k+3}\left\|p_{l}\left(\psi_{n} f\right)\right\|_{L^{2}}^{2}+\left\|p_{k}\left(\psi_{j} f\right)\right\|_{L^{2}}^{2}\right]
\end{gathered}
$$

\subsubsection{Remaining estimates}

In this subsection, we shall use Cauchy Schwarz and Young's inequalities several times to estimate the remaining terms. Firstly, integrating by parts, the second integral of (3.24) becomes

$$
\begin{aligned}
-\int_{v}\left(\bar{a} \nabla \psi_{j}\right) \nabla p_{k}\left(\psi_{m} f\right) p_{k}\left(\psi_{j} f\right) d v & = \\
\int_{v}\left(\bar{a} \nabla \psi_{j}\right) p_{k}\left(\psi_{m} f\right) \nabla p_{k}\left(\psi_{j} f\right) d v & +\int_{v} \nabla\left(\bar{a} \nabla \psi_{j}\right) p_{k}\left(\psi_{m} f\right) p_{k}\left(\psi_{j} f\right) d v .
\end{aligned}
$$

For the second integrals of respectively (3.57), (3.23), (3.25) and (3.26), thanks to inequalities (3.30), we obtain by Young's inequality

$$
\begin{gathered}
\left|\int_{v} \nabla\left(\bar{a} \nabla \psi_{j}\right) p_{k}\left(\psi_{m} f\right) p_{k}\left(\psi_{j} f\right) d v\right| \leq C \mathcal{C}_{j}^{\frac{\gamma}{2}}\left[\left\|p_{k}\left(\psi_{m} f\right)\right\|_{L^{2}}^{2}+\left\|p_{k}\left(\psi_{j} f\right)\right\|_{L^{2}}^{2}\right], \\
\frac{1}{2}\left|\int_{v}\left(\bar{a} \nabla^{2} \tilde{\psi}_{j}+2 \bar{b} \nabla \tilde{\psi}_{j}\right) p_{k}\left(\psi_{j} f\right) p_{k}\left(\psi_{j} f\right) d v\right| \leq C \mathcal{C}_{j}^{\frac{\gamma}{2}}\left\|p_{k}\left(\psi_{j} f\right)\right\|_{L^{2}}^{2},
\end{gathered}
$$




$$
\begin{aligned}
& \frac{1}{2}\left|\int_{v}\left(\bar{a} \nabla^{2} \psi_{j}+2 \bar{b} \nabla \psi_{j}\right) p_{k}\left(\psi_{m} f\right) p_{k}\left(\psi_{j} f\right) d v\right| \leq C \mathcal{C}_{j}^{\frac{\gamma}{2}}\left[\left\|p_{k}\left(\psi_{m} f\right)\right\|_{L^{2}}^{2}+\left\|p_{k}\left(\psi_{j} f\right)\right\|_{L^{2}}^{2}\right] \\
& \frac{1}{2}\left|\int_{v} \bar{a} \nabla \psi_{j} \nabla \psi_{m} p_{k}\left(\psi_{n} f\right) p_{k}\left(\psi_{j} f\right) d v\right| \leq C \mathcal{C}_{j}^{\frac{\gamma}{2}}\left[\left\|p_{k}\left(\psi_{n} f\right)\right\|_{L^{2}}^{2}+\left\|p_{k}\left(\psi_{j} f\right)\right\|_{L^{2}}^{2}\right] .
\end{aligned}
$$

Next, for the first integral of (3.57), using Cauchy Schwarz inequality, we obtain

$$
\left|\int_{v}\left(\bar{a} \nabla \psi_{j}\right) p_{k}\left(\psi_{m} f\right) \nabla p_{k}\left(\psi_{j} f\right) d v\right| \leq C \mathcal{C}_{j}^{\frac{\gamma+1}{2}}\left\|p_{k}\left(\psi_{m} f\right)\right\|_{L^{2}}\left\|\nabla p_{k}\left(\psi_{j} f\right)\right\|_{L^{2}} .
$$

For any constant $\zeta>1$, thanks to Young inequality, the term (3.62) becomes

$$
\frac{C \mathcal{C}_{j}^{\frac{\gamma+1}{2}}}{\zeta}\left\|p_{k}\left(\psi_{m} f\right)\right\|_{L^{2}} \zeta\left\|\nabla p_{k}\left(\psi_{j} f\right)\right\|_{L^{2}} \leq \frac{C \mathcal{C}_{j}^{\gamma+1}}{2 \zeta^{2}}\left\|p_{k}\left(\psi_{m} f\right)\right\|_{L^{2}}^{2}+\frac{\zeta^{2}}{2}\left\|\nabla p_{k}\left(\psi_{j} f\right)\right\|_{L^{2}}^{2} .
$$

Finally, it remains to estimate the second right hand side of (3.22), (3.29) and the first integral of (3.27). Thanks to inequalities (3.30), we obtain that these terms have the same following upper bounds

$$
C \mathcal{C}_{j}^{\frac{\gamma+1}{2}}\left\|p_{k}\left(\psi_{j} f\right)\right\|_{L^{2}}\left\|\nabla p_{k}\left(\psi_{j} f\right)\right\|_{L^{2}}
$$

For any constant $\mu>1$, thanks Young inequality, (3.64) becomes

$$
\frac{C \mathcal{C}_{j}^{\frac{\gamma+1}{2}}}{\mu}\left\|p_{k}\left(\psi_{j} f\right)\right\|_{L^{2}} \mu\left\|\nabla p_{k}\left(\psi_{j} f\right)\right\|_{L^{2}} \leq \frac{C \mathcal{C}_{j}^{\gamma+1}}{2 \mu^{2}}\left\|p_{k}\left(\psi_{j} f\right)\right\|_{L^{2}}^{2}+\frac{\mu^{2}}{2}\left\|\nabla p_{k}\left(\psi_{j} f\right)\right\|_{L^{2}}^{2} .
$$

\subsection{Obtaining a differential equation}

From Bernstein's inequality (2.12), we first note that one has

$$
\left\|p_{k}\left(\psi_{j} f\right)\right\|_{L^{2}} \leq C 2^{\frac{N k}{2}}|| p_{k}\left(\psi_{j} f\right) \|_{L^{1}}
$$

where $\mathrm{C}$ is a non negative constant independent of $\mathrm{k}$ and $\mathrm{j}$.

This holds true for all integers $k \geq 0, j \geq 0$.

Beginning with the terms (3.37), (3.40), (3.47), (3.49), (3.51), (3.53) and (3.55), we get the bound

$$
\leq C \frac{\mathcal{C}_{j}^{\frac{\gamma}{2}}}{2^{(r-1) k}} 2^{\frac{N k}{2}}\left\|p_{k}\left(\psi_{j} f\right)\right\|_{L^{2}}
$$

where $C$ depends on $K,\|\psi\|_{L^{\infty}}$ and $\|f\|_{L^{1}}$.

Then, choosing $\varepsilon=\eta=\zeta=\mu=\frac{\mathcal{C}_{j}^{\frac{\gamma}{4}}}{2}$, gluing together the estimates of the terms (3.39), 
(3.42), (3.48), (3.50), (3.52), (3.54), (3.56), (3.58), (3.59), (3.60), (3.61), (3.63), (3.65) and summing with respect to the exponent of $\mathcal{C}_{j}$, we get the following differential inequality, for all integer $k, j$

$$
\left\{\begin{array}{l}
\partial_{t}\left\|p_{k}\left(\psi_{j} f\right)\right\|_{L^{2}}^{2}+\mathcal{C}_{j}^{\frac{\gamma}{2}}\left\|\nabla p_{k}\left(\psi_{j} f\right)\right\|_{L^{2}}^{2} \leq C \frac{\mathcal{C}_{j}^{\frac{\gamma}{2}}}{2^{(r-1) k}} 2^{\frac{N k}{2}}\left\|p_{k}\left(\psi_{j} f\right)\right\|_{L^{2}} \\
+C \mathcal{C}_{j}^{\frac{\gamma+2}{2}}\left\{\left\|p_{k}\left(\psi_{j} f\right)\right\|_{L^{2}}^{2}+\sum_{l=k-3}^{k+3} \sum_{h=j-2, h \neq j \text { or } l \neq k}^{j+2}\left\|p_{l}\left(\psi_{h} f\right)\right\|_{L^{2}}^{2}\right\},
\end{array}\right.
$$

Dividing (3.68) by $2^{k(N+1)}$, using (2.12), we get a chain of inequalities of the form, for all integers $k \geq 0, j \geq 0$

$$
\partial_{t} U_{k j}(t)+C_{k j} U_{k j}(t) \leq \mathcal{K}_{k j}^{r}(t)+C \mathcal{C}_{j}^{\frac{\gamma+2}{2}}\left\{U_{k j}(t)+\sum_{l=k-3}^{k+3} \sum_{h=j-2, h \neq j \text { or } l \neq k}^{j+2} U_{l h}(t)\right\},
$$

where

$$
\left\{\begin{array}{l}
U_{k j}=\frac{\left\|p_{k}\left(\psi_{j} f\right)\right\|_{L^{2}}^{2}}{2^{k(N+1)}}, C_{k j}=C \mathcal{C}_{j}^{\frac{\gamma}{2}} 2^{2 k}, \mathcal{C}_{j}=\left(1+2^{2 j}\right) \\
\text { and } \mathcal{K}_{k j}^{r}(t)=C \frac{\mathcal{C}_{j}^{\frac{\gamma}{2}}}{2^{r k}}\left\|p_{k}\left(\psi_{j} f\right)(t)\right\|_{L^{1}}
\end{array}\right.
$$

\subsection{Conclusion and regularity}

\section{a) Smoothness with respect to the variable $v$.}

For the estimation of (3.69), we use the iteration method to obtain the regularity in the Schwartz space. Integrating this formula between times $t_{0}$ and $t$, we get

$$
\begin{gathered}
U_{k j}(t) \leq U_{k j}\left(t_{0}\right) e^{-C_{k j} t} \\
+\int_{t_{0}}^{t} e^{C_{k j}(s-t)}\left[\mathcal{K}_{k j}^{r}(s)+\mathcal{C}_{j}^{\frac{\gamma+2}{2}}\left\{U_{k j}(s)+\sum_{l=k-3}^{k+3} \sum_{h=j-2, h \neq j \text { or } l \neq k}^{j+2} U_{l h}(s)\right\}\right] .
\end{gathered}
$$

Next, we fix any non negative variable $\beta<1$, getting an inegality $e^{C_{k j}(s-t)}<\frac{1}{C_{k j}^{\beta}(s-t)^{\beta}}$. Then, we multiply (3.70) by the constant $C_{k j}^{\beta}$ and we sum on the variables $k, j$. Thanks to the inequality (3.66), giving the link with $L^{1}$ space, and the hypothesis (1.7) of the conservation of all moments of $f$, we have, for any integer $r>0$ and for all $t_{0}>0$

$$
\sum_{k=0}^{\infty} \sum_{j=0}^{\infty} C_{k j}^{\beta} U_{k j}(t)<C_{t_{0}}
$$

where $C_{t_{0}}$ is a constant depending only on $K,\|f\|_{L_{\gamma}^{1}}$ and $\|f\|_{L_{\frac{\gamma+2}{2}}^{1}}^{2}$.

Now, we whall show that the series $\sum_{k=0}^{\infty} \sum_{j=0}^{\infty} C_{k j}^{q \beta} U_{k j}(t)$ is finite, for all integer $q$, as soon 
as $t \geq t_{0}$ by iteration over the variable $q$. The previous estimations prove that is true for $q=1$.

Assuming that it is true up to $q$, using inequality (3.66) and the conservation of moments of $f$, we find that, after $q+1$ iterations, for $r \geq 2 q \beta$

$$
\sum_{k=0}^{\infty} \sum_{j=0}^{\infty} C_{k j}^{(q+1) \beta} U_{k j}(t)<C_{t_{0}}
$$

where $C_{t_{0}}$ is a constant depending only on $K,\|f\|_{L_{\gamma+q \beta \gamma}^{1}}$ and $\|f\|_{L_{\frac{\gamma+2+\gamma q \beta}{2}}^{1}}^{2}$.

Hence, we conclude that $\sum_{k=0}^{\infty} \sum_{j=0}^{\infty} C_{k j}^{q \beta} U_{k j}(t)$ is convergent.

In view of Lemma $(2.1), f(t)$ lies in $H_{q \gamma}^{2 q \beta-N-1}$ as soon as $t>0$ and for any integers $q$. In other words, $f \in H_{s}^{h}$ for all $h>0, s \geq \frac{\gamma}{2 \beta}(h+N+1)$.

Finally, all in all, we see that (still when $t>0) f$ lies $H_{s}^{h}$ for all $h, s>0$, and therefore lies in $\mathcal{S}$.

\section{b) Smoothness with respect to the time $t$.}

It remains to show that the previous regularity of the solution $f$ holds true for all derivatives on the variable $t$ i.e $\partial_{t}^{p} f(t) \in \mathcal{S}$ for all integer $p$ as soon as $t>0$, by iterating over the variable $p$, which is already clearly true for $p=0$, in view of the previous step. Next, assuming that it is true on $p$, then for $p+1$

$$
\partial_{t}^{p+1} f=\sum_{k=0}^{p} C_{p}^{k}\left(\partial_{t}^{k} \bar{a}\right) \nabla^{2}\left(\partial_{t}^{p-k} f\right)-\sum_{k=0}^{p} C_{p}^{k}\left(\partial_{t}^{k} \bar{c}\right) \partial_{t}^{p-k} f .
$$

Thanks to the convolution structure, the derivatives of $\bar{a}$ and $\bar{c}$ can be done directly on $f$. Since the derivatives of $f$ of order less or equal to $p$ are in Schwartz space, if we derive (3.71) w.r.t. variable $v$ for any order, and multiply the results by any polynomial, it is still bounded.

Therefore, this implies that the 1.h.s. of (3.71) lies in Schwartz space and concludes the proof of Theorem 1.1, under assumption (1.4).

\section{Proof Theorem 1.1, under assumption (1.5)}

In this section, we consider the hard potentiel case $\chi(|v|)=|v|^{\gamma}$, which has a singularity around zero.

The method of proof used above still applies for this case, up to some modifications that we now explain. 
Firstly, looking at subsection 3.2, the lower bound therein holds true for hard potentiel kernels, as proven in the work of Desvillettes and Villani [9] (Proposition 4).

Some modifications will then appear starting from subsection 3.3, where we need to estimate from above different terms using the commutator iteration method (that is, Lemmas 3.1 and 3.2 ), and this is really here that smoothness of the kernel is needed.

In order to get ride of the singularity near 0, during this subsection, we shall split the kernel into two parts. The first one will be smooth, thus avoiding the singularity and therefore we can apply to it the manipulations of the iteration's method. For the second one, we shall show that it is possible to make it as small as one wishes, in a suitable sense.

In the sequel, as a convention, we shall use subscripts S for "smooth" and R for "remainder". This type of decomposition is used several times in [16], but is standard.

Having in mind subsection 3.3, we proceed as follows.

Firstly, we take $\Phi: \mathbb{R} \longrightarrow \mathbb{R}_{+}$an even $C^{\infty}$ function such that supp $\Phi \subset(-1,1)$, being 1 near 0 . Then, we write, for any strictly non negative constant $\alpha_{k}$ depending on $k$,

$$
\chi=\chi^{S, k}+\chi^{R, k}, \quad \chi^{S, k}=|v|^{\gamma} \bar{\Phi}_{\alpha_{k}}, \quad \chi^{R, k}=|v|^{\gamma} \Phi_{\alpha_{k}}
$$

where $\bar{\Phi}_{\alpha_{k}}=1-\Phi_{\alpha_{k}}$ such that $\Phi_{\alpha_{k}}(|v|)=\Phi\left(\frac{|v|}{\alpha_{k}}\right)$ for all $v \in \mathbb{R}^{n}$.

After some computations, we note that different derivatives of the smooth part gives, for any integer $\beta$, the bound

$$
\left|\nabla^{\beta} \chi^{S, k}(|v|)\right| \leq C_{\beta}|v|^{\gamma-\beta} \bar{\Phi}_{\alpha_{k}},
$$

for a non negative constant $C_{\beta}$ depending only on $\beta$.

We write, with clear notations

$$
a=a^{S, k}+a^{R, k}, b=b^{S, k}+b^{R, k}, c=c^{S, k}+c^{R, k} .
$$

In view of the convolution structure, taking (4.72) into account, one has, for all $v \in \mathbb{R}^{N}$

$$
\begin{gathered}
\left|\bar{a}^{S, k}(v)\right| \leq C|v|^{\gamma+2} \bar{\Phi}_{\alpha_{k}}, \quad\left|\bar{b}^{S, k}(v)\right| \leq C|v|^{\gamma+1} \bar{\Phi}_{\alpha_{k}}, \\
\left|\nabla^{\beta} \bar{a}^{S, k}(v)\right| \leq C C_{\beta}|v|^{\gamma+2-\beta} \bar{\Phi}_{\alpha_{k}} \quad \forall \beta \geq 2,
\end{gathered}
$$

for a constant $\mathrm{C}$ depending here only on $\|f\|_{L_{\gamma+2}^{1}}$.

Therefore, we obtain, using the precise support sets of $\psi_{j}$ and $\tilde{\psi}_{j}$, the following inequalities, for all $j \in \mathbb{N}$

$$
\left\{\begin{array}{l}
\left|\bar{a}^{S, k} \psi_{j}\right| \leq C 2^{j(\gamma+2)},\left|\bar{a}^{S, k} \nabla \psi_{j}\right| \leq C 2^{j(\gamma+1)},\left|\bar{a}^{S, k} \nabla^{2} \psi_{j}\right| \leq C 2^{j \gamma} \\
\left|\bar{b}^{S, k} \psi_{j}\right| \leq C 2^{j(\gamma+1)},\left|\bar{b}^{S, k} \nabla \psi_{j}\right| \leq C 2^{j \gamma} \\
\text { and }\left|\nabla^{\beta}\left(\bar{a}^{S, k} \psi_{j}\right)\right| \leq C C_{\beta} 2^{j \gamma}, \quad \forall \beta \geq 2
\end{array}\right.
$$


for a constant $\mathrm{C}$ depending on $\|f\|_{L_{\gamma+2}^{1}}$ and the $L^{\infty}$ bound of $\psi$ and its derivatives. Of course, these inequalities also hold true if we change $\psi_{j}$ by $\tilde{\psi}_{j}$.

For the non smooth part, taking into account the support of $\Phi_{\alpha_{k}}$, we obtain the following bounds

$$
\left|\bar{a}^{R, k}(v)\right| \leq C \alpha_{k}^{\gamma+2}, \quad\left|\bar{b}^{R, k}(v)\right| \leq C \alpha_{k}^{\gamma+1},\left|\bar{c}^{R, k}(v)\right| \leq C \alpha_{k}^{\gamma},
$$

for a constant $\mathrm{C}$ depending on $\|f\|_{L^{1}}$.

Next, after decomposition of the kernel, as regards to the estimates of the integrals in subsection 3.3.2, we are reduced to treat two types of integrals, corresponding respectively to the smooth and non smooth parts.

For the smooth ones, taking into account estimates (4.74), the same estimations clearly hold true again.

Concerning the non smooth integrals, we shall use estimates (4.75), to obtain different upper bounds. For instance, using Taylor's expansion at order 1, we obtain

$$
\begin{aligned}
\left|\int_{v}\left[\nabla^{2} p_{k}, \bar{a}^{R, k} \tilde{\psi}_{j}\right] f p_{k} f d v\right| & \leq\left\|\left[\nabla^{2} p_{k}, \bar{a}^{R, k} \tilde{\psi}_{j}\right] f\right\|_{L^{1}}\left\|p_{k} f\right\|_{L^{\infty}} \quad \text { (Holder inequality) } \\
& \leq C 2^{k} \alpha_{k}^{\gamma+1}\|f\|_{L^{1}} 2^{\frac{N k}{2}}\left\|p_{k} f\right\|_{L^{2}} \quad \text { (Bernstein inequality) } \\
& \leq C 2^{k} \alpha_{k}^{\gamma+1}\|f\|_{L^{1}} 2^{\frac{N k}{2}}\left\|p_{k} f\right\|_{L^{2}}
\end{aligned}
$$

In the same way, we have also the two estimates

$$
\begin{aligned}
\left|\int_{v}\left[\nabla p_{k}, \bar{b}^{R, k} \tilde{\psi}_{j}\right] f p_{k} f d v\right| & \leq\left\|\left[\nabla p_{k}, \bar{b}^{R, k} \tilde{\psi}_{j}\right] f\right\|_{L^{1}}\left\|p_{k} f\right\|_{L^{\infty}} \quad \text { (Holder inequality) } \\
& \leq C \alpha_{k}^{\gamma}\|f\|_{L^{1}} 2^{\frac{N k}{2}}\left\|p_{k} f\right\|_{L^{2}} \quad \text { (Bernstein inequality) } \\
& \leq C \alpha_{k}^{\gamma}\|f\|_{L^{1}} 2^{\frac{N k}{2}}\left\|p_{k} f\right\|_{L^{2}}
\end{aligned}
$$

and

$$
\begin{aligned}
\left|\int_{v}\left[p_{k}, \bar{b}^{R, k} \nabla \tilde{\psi}_{j}\right] f p_{k} f d v\right| & \leq\left\|\left[p_{k}, \bar{b}^{R, k} \nabla \tilde{\psi}_{j}\right] f\right\|_{L^{1}}\left\|p_{k} f\right\|_{L^{\infty}} \quad \text { (Holder inequality) } \\
& \leq C \alpha_{k}^{\gamma}\|f\|_{L^{1}} 2^{\frac{N k}{2}}\left\|p_{k} f\right\|_{L^{2}} \quad \text { (Bernstein inequality) } \\
& \leq C \alpha_{k}^{\gamma}\|f\|_{L^{1}} 2^{\frac{N k}{2}}\left\|p_{k} f\right\|_{L^{2}} .
\end{aligned}
$$

Concerning subsection 3.3.3, the above splitting is not needed, and thus the bounds therein remain true, replacing $\mathcal{C}_{j}^{\frac{1}{2}}$ by $2^{j}$. 
All in all, we get the following slightly different inequality, for $\alpha_{k}<1$,

$$
\left\{\begin{array}{l}
\partial_{t}\left\|p_{k}\left(\psi_{j} f\right)\right\|_{L^{2}}^{2}+2^{j \gamma}\left\|\nabla p_{k}\left(\psi_{j} f\right)\right\|_{L^{2}}^{2} \leq C \frac{2^{j \gamma}}{2^{(r-1) k}} 2^{\frac{N k}{2}}\left\|p_{k}\left(\psi_{j} f\right)\right\|_{L^{2}}+C 2^{k} \alpha_{k}^{\gamma} 2^{\frac{N k}{2}}\left\|p_{k}\left(\psi_{j} f\right)\right\|_{L^{2}} \\
+C 2^{j(\gamma+2)}\left\{\left\|p_{k}\left(\psi_{j} f\right)\right\|_{L^{2}}^{2}+\sum_{l=k-3}^{k+3} \sum_{h=j-2, h \neq j \text { or } l \neq k}^{j+2}\left\|p_{l}\left(\psi_{h} f\right)\right\|_{L^{2}}^{2}\right\} .
\end{array}\right.
$$

Dividing (4.76) by $2^{k(N+1)}$, using (2.12), we get a chain of inequalities of the form, for any integers $k, j$

$\partial_{t} U_{k j}(t)+C_{k j} U_{k j}(t) \leq \mathcal{K}_{k j}^{r}(t)+\mathcal{H}_{k j}(t)+C 2^{j(\gamma+2)}\left\{U_{k j}(t)+\sum_{l=k-3}^{k+3} \sum_{h=j-2, h \neq j \text { or } l \neq k}^{j+2} U_{l h}(t)\right\}$,

where

$$
\left\{\begin{array}{l}
U_{k j}=\frac{\left\|p_{k}\left(\psi_{j} f\right)\right\|_{L^{2}}^{2}}{2^{k(N+1)}}, C_{k j}=C 2^{j \gamma} 2^{2 k}, \mathcal{K}_{k j}^{r}(t)=C \frac{2^{j \gamma}}{2^{r k}}\left\|p_{k}\left(\psi_{j} f\right)(t)\right\|_{L^{1}} \\
\text { and } \mathcal{H}_{k j}(t)=C \alpha_{k}^{\gamma}\left\|p_{k}\left(\psi_{j} f\right)(t)\right\|_{L^{1}} .
\end{array}\right.
$$

To conclude with the regularity, we proceed as in subsection 3.5. We obtain the same inequality but with one more term, depending on $\alpha_{k}$.

To get the convergence of the series, it is enough to take $\alpha_{k}=\frac{1}{2^{k \kappa}}$ for a suitable $\kappa>0$. In

particular, for our case, it suffices to choose $\kappa$ large enough w.r.t. $\frac{2 q \beta+1}{\gamma}$ for any fixed integer $q$ (see subsection 3.5).

\section{Appendix}

Definition 4.1 Let $k \in \mathbb{N}$. We denote $p_{k}$ and $\tilde{p}_{k}$ the Littlewood-Paley operators defined in section 2. These operators can be written under the following convolution form, for $f \in L^{1}$

- $p_{k} f=\phi_{k} * f$ with $\phi_{k}()=.2^{N k} \hat{\psi}\left(2^{k}\right.$.) with $\phi_{k} \in \mathcal{S}$

- $\tilde{p}_{k} f=\tilde{\phi}_{k} * f$ with $\tilde{\phi}_{k}()=.2^{N k} \hat{\tilde{\psi}}\left(2^{k}\right.$. $)$ with $\tilde{\phi}_{k} \in \mathcal{S}$,

where $\psi$ and $\tilde{\psi}$ are the smooth functions given in section 2.

Proposition 4.1 Let $k \in \mathbb{N}, r \in \mathbb{N}^{*}$ and $h=0,1,2$. Let $\varphi$ be any smooth bounded function together with its derivatives. Define the linear commutator operator

$$
\tilde{T}_{k}^{r, h}=\overbrace{\left[\tilde{p}_{k},\left[\tilde{p}_{k},\left[\tilde{p}_{k}, \cdots\right.\right.\right.}^{r},\left[\nabla^{h} p_{k}, \varphi\right]]]] .
$$

Then, there exists a constant number $C>0$ such that, for all $1 \leq p \leq \infty$,

$$
\left\|\tilde{T}_{k}^{r, h}\right\|_{L^{p} \rightarrow L^{p}} \leq \frac{C C_{r}}{2^{(r+1-h) k}} \text { where } C_{r}=\left\|\nabla^{r+1} \varphi\right\|_{L^{\infty}} .
$$


Proof: Using Taylor's expansion of $\varphi$ on $v$ until order $r+1$, we get, for all $v, v_{*} \in \mathbb{R}^{N}$ $\varphi\left(v^{*}\right)=\varphi(v)+\frac{\left(v^{*}-v\right)}{1 !} \nabla \varphi(v)+\frac{\left(v^{*}-v\right)^{2}}{2 !} \nabla^{2} \varphi(v)+\cdots \cdots+\frac{\left(v^{*}-v\right)^{r+1}}{(r+1) !} \nabla^{r+1} \varphi(c)$ with $c \in\left[v^{*}, v\right]$. So, we can write, for $u \in L^{p}\left(\mathbb{R}^{N}\right)$

$$
\begin{aligned}
{\left[\nabla^{h} p_{k}, \varphi\right](u) } & =\int_{v^{*}} \nabla^{h} \phi_{k}\left(.-v^{*}\right)\left[\varphi\left(v^{*}\right)-\varphi(.)\right] u\left(v^{*}\right) d v^{*} \\
& =\sum_{m=1}^{r} \Theta_{k}^{m, h}(u)+\Gamma_{k}^{r+1, h}(u),
\end{aligned}
$$

where

$$
\left\{\begin{array}{l}
\Theta_{k}^{m, h}(u)=\frac{1}{m !} \nabla^{m} \varphi\left[\rho_{k}^{m, h} * u\right] \\
\Gamma_{k}^{r+1, h}(u)(v)=\frac{1}{(r+1) !} \int_{v^{*}} \rho_{k}^{r+1}\left(v-v^{*}\right) \nabla^{r+1} \varphi(c) u\left(v^{*}\right) d v^{*} \text { and } \rho_{k}^{m, h}=(-1)^{m} v^{m} \nabla^{h} \phi_{k}(.) .
\end{array}\right.
$$

We simplify the notations to write $\tilde{T}_{k}^{r, h}$ under the form

$$
\begin{aligned}
\tilde{T}_{k}^{r, h}(u) & =\sum_{m=1}^{r} \overbrace{\left[\tilde{p}_{k},\left[\tilde{p}_{k},\left[\tilde{p}_{k}, \cdots\right.\right.\right.}^{r},\left[\Theta_{k}^{m, h}\right]]]](u) \\
& +\overbrace{\left[\tilde{p}_{k},\left[\tilde{p}_{k},\left[\tilde{p}_{k}, \cdots\right.\right.\right.}^{r},\left[\Gamma_{k}^{r+1, h}\right]]]](u) .
\end{aligned}
$$

Taking the notation $\tilde{\rho}_{k}^{m}=(-1)^{m} v^{m} \tilde{\phi}_{k}($.$) for all integer m$.

After some computations, thanks to Taylor's expansion which we apply several times, we obtain

$$
\begin{aligned}
& \tilde{T}_{k}^{r}(u)(v)=\sum_{m=1}^{r} \sum_{i_{1}+i_{2}+\cdots+i_{r-m+1}=r-m+1} \int_{x_{1}} \cdots \int_{x_{m}} \cdots \int_{x_{r}} \int_{v^{*}} d x_{1} \cdots d x_{r} d v^{*} \frac{1}{i_{1} ! i_{2} ! \cdots i_{r-m+1} !} \\
& \tilde{\phi}_{k}\left(v-x_{1}\right) \cdots \tilde{\phi}_{k}\left(x_{m-2}-x_{m-1}\right) \tilde{\rho}_{k}^{i_{1}}\left(x_{m-1}-x_{m}\right) \cdots \tilde{\rho}_{k}^{i_{r-m+1}}\left(x_{r-1}-x_{r}\right) \rho_{k}^{m, h}\left(x_{r}-v^{*}\right) \nabla^{r+1} \varphi(c) u\left(v^{*}\right) \\
& +\frac{1}{(r+1) !} \int_{x_{1}} \cdots \int_{x_{r}} \int_{v^{*}} d x_{1} \cdots d x_{r} d v^{*} \tilde{\phi}_{k}\left(v-x_{1}\right) \cdots \tilde{\phi}_{k}\left(x_{r-1}-x_{r}\right) \rho_{k}^{r+1, h}\left(x_{r}-v^{*}\right) \nabla^{r+1} \varphi\left(c^{\prime}\right) u\left(v^{*}\right)
\end{aligned}
$$

where $c, c^{\prime} \in\left[v, x_{1} \cdots x_{r}\right],\left(i_{1}, i_{2}, \cdots, i_{r}\right) \in \mathbb{N}^{r}$.

So,

$$
\left\{\begin{array}{c}
\left|\tilde{T}_{k}^{r, h}(u)\right| \leq C_{r} \sum_{m=1}^{r} \sum_{i_{1}+i_{2}+\cdots+i_{r-m+1}=r-m+1}^{r} \overbrace{\left|\tilde{\phi}_{k}\right| * \cdots *\left|\tilde{\phi}_{k}\right|}^{m-1} *\left|\tilde{\rho}_{k}^{i_{1}}\right| * \cdots *\left|\tilde{\rho}_{k}^{i_{r-m+1}}\right| *\left|\rho_{k}^{m, h}\right| *|u| \\
+C_{r} \overbrace{\tilde{\phi}_{k}|* \cdots *| \tilde{\phi}_{k} \mid}^{m-1} *\left|\rho_{k}^{r+1, h}\right| *|u|,
\end{array}\right.
$$


where $C_{r}=C\left\|\nabla^{r+1} \varphi\right\|_{L^{\infty}}$.

Since $\hat{\psi}, \hat{\tilde{\psi}} \in \mathcal{S}$, we get, for all integer $m$

$$
\left\{\begin{array}{l}
\left\|\rho_{k}^{m, h}\right\|_{L^{1}} \leq \frac{c_{m, h}}{2^{k(m-h)}} \text { where } c_{m, h}=\left\||v|^{m} \nabla^{h} \hat{\psi}\right\|_{L^{1}} \\
\left\|\tilde{\phi}_{k}\right\|_{L^{1}} \leq \tilde{c}_{0}, \quad\left\|\tilde{\rho}_{k}^{m}\right\|_{L^{1}} \leq \frac{\tilde{c}_{m}}{2^{k m}} \text { where } \tilde{c}_{m}=\left\||v|^{m} \hat{\tilde{\psi}}\right\|_{L^{1}}
\end{array}\right.
$$

Thanks to estimates (4.77) and (4.78), there exists a strictly non negative constant $C$, depending on $c_{m, h}, \tilde{c}_{m}(1 \leq m \leq r)$, such that, for all $1 \leq p \leq \infty$

$$
\left\|\tilde{T}_{k}^{r, h}\right\|_{L^{p} \rightarrow L^{p}} \leq \frac{C C_{r}}{2^{(r+1-h) k}} .
$$

Acknowledgments: I would like to thank R.Alexandre for many helpful discussions during the preparation of this work.

\section{References}

[1] A.A.Arsen'ev, O.E.Buryak On the connection between a solution of the Boltzmann equation and a solution of the Landau-Fokker-Plank equation. Math.USSR Sbornik, 69 (2), 465-478, 1991.

[2] R.AleXANDRE Integral kernel estimates for a linear singular operator linked with Boltzmann equation. Part I: Small singularities $0<\nu<1$. Preprint.

[3] R.Alexandre, M.ElSafadi Littlewood-Paley decomposition and regularity issues in Boltzmann homogeneous equations.I. Non cutoff and Maxwell cases. M3AS, 15 (6), 2005.

[4] R.Alexandre, M.ElSafadi Littlewood-Paley decomposition and regularity issues in Boltzmann homogeneous equations.II. Non cutoff and non Maxwell cases. work in progress.

[5] R.Alexandre, C.Villani On the Landau approximation in plasma physics. Ann. Inst. H. Poincaré Anal. Non Linéaire, 21 (1), 61-95, 2004.

[6] P.Degond, M.Lemou. Dispersion relations for the linearized Fokker-Plank equation. Arch. Rat. Mech. Anal, 138 (2), 687-702, 1989.

[7] L.Desvillettes On Asymptotics of the Boltzmann Equation when the Collisions Become Grazing. Transport Theory and Statistical Physics, 21 (3), 259-276, 1992. 
[8] L.Desvillettes, B.Wennberg Smoothness of the solution of the spatially homogeneous Boltzmann equation without cutoff. Comm. P.D.E, 29 (1-2), 133-155, 2004.

[9] L.Desvillettes, C.Villani On the spatially homogeneous Landau equation for hards potentials.PartI: Existence, uniqueness and smoothness. Comm. P.D.E, 25 (1/2), 179$259,2000$.

[10] L.Desvillettes, C.Villani On the Spatially Homogeneous Landau Equation for Hard Potentials. Part II: H-Theorem and Applications, Communication in Partial Differential Equations, 25 (1-2), 261-298, 2000.

[11] T.Goudon On Boltzmann equation and Fokker-Planck asymptotics: influence of grazing collisions. J. Stat. Phys, 89 (3-4), 751-776, 1997.

[12] H.Guerin, S.MÉLÉARD Convergence from Boltzmann to Landau with soft potential and particle approximations. J. Statist. Phys, 111 (3/4), 931-966, 2003.

[13] P.LemariÉ.-G Recent developments in the Navier-Stokes problem. Research Notes in Mathematics, Vol. 431, Chapman and Hall/CRC, New-York 2002.

[14] P.L. Lions On Boltzmann equation and Landau equation. Phil. Trans. R. Soc. Lond., A, 346, 191-204, 1994.

[15] E.M.Lifchitz, L.P.Petaevski Kinetic theory. MIR, Moscow, 1979.

[16] C.Mouhot, C.Villani Regularity theory for the spatially homogeneous Boltzmann equation with cut-off. Arch. Rational Mech. Anal, 173 (2), 169-212, 2004.

[17] M.Stein Harmonic Analysis. Real variable methods, orthogonality and oscillatory integrals. Princeton Univ. Press, Princeton 1993.

[18] L.Pareschi, G.Russo, G.Toscani Fast spectral methods for the Fokker-Plank-Landau collision operator. J. Comput. Phys, 156 (1), 216-236, 2000.

[19] T.TAO Littlewood-Paley decomposition. Lecture Notes 254A. See web page http://www.math.ucla.edu/ ${ }^{\sim}$ tao/.

[20] H.Triebel Theory of function spaces. Birkhauser Verlag, Basel and al. 1983.

[21] T.Runst, W.Sickel Sobolev spaces of fractional order, Nemytskij operators and Non linear PdE. De Gruyter, New York 1996. 
[22] C.Villani On a new class of solutions for the spatially homogeneous, Boltzmann and Landau equations. Arch. Rat. Mech. Anal, 143 (3), 273-307, 1998.

[23] C.Villani On the spatially homogeneous Landau equation for Maxwellian molecules. Math. Meth. Mod. Appl. Sci, 8 (6), 957-983, 1998.

[24] C.Villani A review of mathematical topics in collisional kinetic theory. in Handbook of Fluid Mechanics, Ed. S. Friedlander, D.Serre 2002.

[25] C.Villani On the Landau equation: weak stability, global existence. Adv. Diff. Eq, 1 (5), 793-816, 1996. 